\title{
Impact of the Deficiency of Micronutrients on Patients with HIV/Aids
}

\section{Impacto de la Deficiencia de Micronutrientes en Pacientes con VIH/Sida}

\section{J. L. Valdés González¹, U. Solis Cartas ${ }^{1}$, and X. P. Manzano Herdoiza²}

${ }^{1}$ Carrera de Medicina, Facultad de Salud Pública, Escuela Superior Politécnica de Chimborazo, Riobamba, Ecuador

${ }^{2}$ Carrera de Medicina, Facultad de Salud Pública, Universidad Nacional de Chimborazo,

XVIII International Seminar on Health, Food and Human Nutrition

Corresponding Author:

J. L. Valdés González

jorge.valdes@espoch.edu.eco jorval1969@hotmail.com

Published: 9 September 2021

Production and Hosting by

Knowledge E

(c) J. L. Valdés González et al. This article is distributed under the terms of the Creative Commons Attribution License, which permits unrestricted use and redistribution provided that the original author and source are credited.

\section{Abstract}

Introduction: HIV-AIDS is an infectious disease that damages the immune system, putting you at risk of serious infections and certain types of cancer. Micronutrient deficiency varies widely, depending on the population and stage of the disease. It can contribute to the weakening of the immune state and the worsening of the physical condition. Objective: To identify through the collection of nutritional information the consequences produced by the lack of micronutrients in patients with HIV-AIDS. Methodology: It was carried out through bibliographic reviews, scientific articles in order to find true and proven information on the effect that micronutrient deficiency has on people with HIV/AIDS. Results: It is shown that clinical deficiencies of some nutrients occur rapidly in response to dietary deficiencies, malabsorption, or altered metabolism, while those nutrients that have reserves in the body take longer to deplete. Discussion: The acquired immunodeficiency virus that causes AIDS; is associated with weight loss due to malnutrition. There are foods that can counteract many of the symptoms of the patient with HIV/AIDS, hence the importance of their knowledge. Conclusion: Micronutrient deficiency influences the immune system, accentuating the immunodeficiency that leads to AIDS. People living with this disease should have a balanced diet with Vitamins A, B, zinc and iron to strengthen the immune system, these offer a safe and economical treatment to slow the progression of the infection.

Keywords: micronutrients, HIV, AIDS.

\section{Resumen}

Introducción: EI VIH-SIDA es una enfermedad infecciosa que daña el sistema inmunitario lo que pone en riesgo de contraer infecciones graves y ciertos tipos de cáncer. La deficiencia de micronutrientes varía ampliamente, según la población y el estadio de la enfermedad; puede contribuir al debilitamiento del estado inmune y al empeoramiento de la condición física. Objetivo: Identificar mediante la recopilación de información nutricional las consecuencias que produce la falta de micronutrientes en pacientes con VIH-SIDA. Metodología: Se realizó a través de revisiones bibliográficas, artículos científicos con el fin de encontrar información verídica y comprobada del efecto que tiene la deficiencia de micronutrientes en personas con VIH/SIDA. Resultados: Se demuestra que las deficiencias clínicas de algunos nutrientes ocurren rápidamente en respuesta a deficiencias dietéticas, malabsorción, o metabolismo alterado, mientras que aquellos nutrientes que tienen reservas en el cuerpo tardan más en agotarse. Discusión: El virus de la inmunodeficiencia adquirida causante del SIDA; está asociada con la pérdida de peso por malnutrición. Hay muchos alimentos del grupo de 
los micronutrientes que minimizan manifestaciones clínicas en los enfermos el síndrome de inmunodeficiencia adquirida, siendo necesario saber cuáles son. Conclusiones: La deficiencia de micronutrientes influye en el sistema inmune, acentuando la inmunodeficiencia que lleva al SIDA. Las personas que viven con esta enfermedad deben tener una dieta balanceada con Vitaminas A, B, zinc y hierro para fortalecer el sistema inmunológico, estas ofrecen un tratamiento seguro y económico para retardar la progresión de la infección.

Palabras Clave: micronutrientes, VHI, SIDA.

\section{Introducción}

EI SIDA no fue descubierto hasta principios de la década de los 80 cuando médicos estadounidenses empezaron a observar que había grupos de pacientes con enfermedades muy poco comunes. Los primeros casos se detectaron entre homosexuales de Nueva York y California. Estas personas padecían enfermedades como el sarcoma de Kaposi, un tipo raro de cáncer de piel, así como un tipo de infección pulmonar que transmiten los pájaros.

Pronto se detectaron también casos entre drogadictos por vía intravenosa y receptores de transfusiones de sangre. En 1982 se dio nombre a esta enfermedad: Síndrome de inmunodeficiencia adquirida. Desde entonces el SIDA ha matado a unos 25 millones de personas en todo el mundo y ha dejado huérfanos a 12 millones de niños sólo en África [1].

Esta patología es producida por el virus del VIH/SIDA, en cuanto a su mecanismo de producción se conoce que debilita al sistema defensivo del ser humano, produciendo perdida de los elementos que enfrentan las infecciones, los de la serie blanca en la sangre, por tal motivo le pone en peligro de coger alguna enfermedad peligrosa, así como algunas neoplasias.

Entonces la palabra SIDA quiere decir un conjunto de síntomas y signos que destruyen el sistema inmunológico, siendo la fase terminal la enfermedad del $\mathrm{VIH}$, por lo tanto todas los pacientes con esta enfermedad van a desencadenar el SIDA, la causa más frecuente de contagio es por medio del sexo sin condón con otra persona que ya tiene esta enfermedad, también durante la gestación o el alumbramiento se pueden transmitir a los niños, las manifestaciones mas frecuentes en la infancia por esta patología es aparición de adenopatías, y manifestaciones catarrales, que duran de forma intermitente más o menos un mes.

Las manifestaciones clínicas más complicadas se demoran muchos años en presentarse, aunque en ovaciones no es tan así, porque se pueden presentar más rápido, esto está dado a el cuidado de cada paciente, en cuanto a la alimentación, y mantener el sistema inmunológico comenzado, para el diagnostico de esta compleja enfermedad simplemente se hace una prueba en sangre [2].

Esta enfermedad está demostrada que se puede transmitir por el sexo con otra persona infestada, así como por pinchazos con objetos cortopunzantes y de la madre 
al feto si esta en el embarazo, pero no es transmitidas a otras personas por usar objetos personales, ni por tocarlos, como puede ser en otras enfermedades conocidas [3].

Dado a las características de esta enfermedad en este momento sigue floreciendo como una grave emergencia para la humanidad y el sistema medico en todo el mundo, ha producido un sin número de muertes por si sola o por las diferentes complicaciones que produce la misma, así como, cada año hay mas personas infestadas en el mundo [4].

Los micronutrientes incluyen los minerales y las vitaminas. Los más conocidos están, el selenio, zinc, cobre, vitamina A, B y E. sí están bajos en el ser humano interfieren en muchos metabolismos celulares y esto hace que bajen más las defensas de las personas afectadas por $\mathrm{VIH}$, por lo que puede enfermar más rápido con SIDA. Esto no está claro en estos momentos por lo tanto tenemos que dar una nutrición adecuada a estos pacientes con los micronutrientes mas conocidos, de esta forma se puede mantener mas saludables a las personas que están enfermas de $\mathrm{VIH}$ y así podremos retrasar la enfermedad del SIDA.

\section{Metodología}

Se llevó a cabo una búsqueda actualizada en los últimos 5 años, a través de páginas de medicina basada en datos clínicos, revisiones de libros web, libros médicos nutricionales. En la selección de los estudios, se obtuvieron de las búsquedas 50 documentos, de las cuales se escogieron 30 que incluyen revisiones sistemáticas, guías de prácticas clínicas, resúmenes, estructuradas de unos artículos originales y datos clínicos. Se excluyeron los artículos con terapias no incluidas en el tratamiento del VIH/SIDA irrelevantes para nuestro objetivo.

\section{Resultados}

Toda persona diagnosticada con infección por VIH causante del SIDA, requiere inicialmente una evaluación nutricional completa, es decir, individualizada, organizada, continua y concisa, dando la debida importancia a la suministración de una dieta equilibrada, con alimentos y nutrientes que contribuyan al mantenimiento y mejora del estado nutricional, desde el diagnóstico de la enfermedad es vital asegurar una correcta alimentación conservando y/o mejorando el aumento de peso y masa muscular.

Los objetivos de una buena nutrición es aumentar las defensas del organismo permitiendo mantener sus reservas, facilitando la recuperación de infecciones oportunistas; mejorar la calidad de vida, ya que permitirá al paciente sentirse con más fuerza y vitalidad y por último mejorar la tolerancia a la medicación, ya que las carencias nutricionales pueden modificar su liberación y metabolismo, aumentando así la sensibilidad a la toxicidad medicamentosa [5] 


\subsection{Nutrición y estadios de la enfermedad}

El paciente con $\mathrm{VIH}$, pasa por diferentes estadios y situaciones, por lo que se intentara mantener desde el inicio de la infección un adecuado estado nutricional, mediante una alimentación equilibrada ajustada a sus necesidades. Cuando el paciente presenta infecciones oportunistas y/o deterioro inmunológico, la intervención nutricional debe ser más rápida e intensa, a las repercusiones metabólicas de la infección por VIH, añadiendo a esto las alteraciones metabólicas secundarias al TAR y su posible repercusión a largo plazo.

Podemos decir que la intervención nutricional debe iniciarse de forma precoz e individualizada en cada paciente desde el momento del diagnóstico de la infección $\mathrm{VIH}$, dependiendo principalmente de su situación clínica e inmunológica para evitar la aparición de cuadros de malnutrición severa incluyendo de manera directa los tratamientos a los que esté sometido, sus hábitos alimentarios, su situación socioeconómico y estado psicológico [6].

Association of Nutrition Services Agencies (ANSA), considera que no existe pautas claras para la suplementación de nutrientes en la enfermedad VIH. Por ello se recomiendan un suplemento multivitamínico-mineral equilibrado a niveles aproximados del $100 \%$ de las RDA. También añade que el empleo de dosis elevadas de vitaminas y minerales debe desaconsejarse, ya que ello puede intensificar alteraciones gastrointestinales previas o anorexia. Asimismo, algunas pueden ser tóxicas a dosis elevadas, caso de las vitaminas A, B6, D, cobre, hierro, niacina, selenio y zinc [7].

\subsection{Nutrición y el sistema inmune}

Las deficiencias de macro y micro nutrientes se asocian con una respuesta inmunológica inadecuada, incrementando el progreso de la enfermedad, aumentando la mortalidad y el riesgo de contraer infecciones oportunistas. Las deficiencias de nutrientes se deben a problemas de mal absorción, anorexia inducida por los medicamentos, las deficiencias nutricionales pueden menoscabar los progresos alcanzados por las Terapias Antirretrovirales Altamente Activas (HAART) [8].

En los pacientes con VIH/SIDA, la presencia de desnutrición predice fuertemente la sobrevida de éstos independientemente de la cuenta de linfocitos T CD4+. Deficiencias clínicas de algunos nutrientes ocurren rápidamente en respuesta a deficiencias dietéticas, malabsorción, o metabolismo alterado, mientras que aquellos nutrientes que tienen reservas en el cuerpo tardan más en agotarse.

A pesar del progreso tan importante que se ha realizado en el campo de la infección por VIH/SIDA, aún es muy grande el desconocimiento en lo relativo a los mecanismos por medio de los cuales el virus de la inmunodeficiencia humana afecta la nutrición, en especial a los pacientes pediátricos y adolescentes. Mayores esfuerzos se deben de dirigir a caracterizar la composición corporal conforme la infección por VIH/SIDA progresa, permitiendo implementar intervenciones nutricionales apropiadas [9].

Según las investigaciones realizadas corroboran que: El retinol o vitamina A es capaz de suprimir la replicación del VIH al inhibir su transcripción; esto ocurre debido a ciertas 
proteínas específicas del retinol, las cuales se ligan a la cápsula viral y previenen la trascripción de la proteína viral, la cual es indispensable para la replicación. Esta supresión de la replicación viral disminuye la cantidad de virus circulante y reduce la habilidad del virus para causar enfermedad; además, lentifica la progresión de la enfermedad y, posiblemente, disminuye el riesgo de morbilidad [10, 11].

Vitamina E. El VIH, y las enfermedades oportunistas, pueden promover el aumento de los niveles de los radicales libres y el estrés oxidativo. La presencia de estos radicales libres se ha relacionado con una progresión acelerada hacia el sida [12].

Estudios sobre aporte complementario de vitamina E. La mayoría de las investigaciones con vitamina $\mathrm{E}$ se han hecho en ratones infectados con $\mathrm{VIH}$, las cuales han demostrado una normalización de los parámetros inmunes, como el aumento de la actividad de las células NK, la disminución de la IL-6, del FNT, el aumento de los Th1 y la disminución de la peroxidación lipídica en el hígado [13].

Selenio: La deficiencia de selenio está asociada con la disminución de la glutatión per oxidasa (GPX), al aumentar los niveles de los radicales libres disponibles y estimular la replicación del VIH y la apoptosis de los linfocitos T CD4; además, esta deficiencia acentúa la inmunodeficiencia del sida, lo cual contribuye a la patogenicidad de varios virus [14, 15]. Zinc: Es un micronutriente esencial, cuya deficiencia está asociada con el desarrollo de inmunosupresión. Esta deficiencia conduce a un aumento en la susceptibilidad a las infecciones, aumento en la replicación del VIH, daño en la inmunidad celular, aceleración de la apoptosis de las células involucradas en la respuesta inmune, disminución de los linfocitos T CD4 y aumento de la carga viral y de la mortalidad $[16,17]$.

Vitaminas del complejo B: La deficiencia de vitaminas del complejo B lleva a una disfunción del sistema inmune. Se producen anticuerpos de las células parietales gástricas, se acelera la progresión de la enfermedad, se disminuye el recuento de linfocitos T CD4, puede contribuir al complejo demencial asociado a sida y, en algunos casos, está relacionado con la neuropatía periférica y la mielopatía [12].

En la mayoría de estudios no arrojan datos exactos sobre la relación de micronutrientes en la enfermedad inmunitaria. Sin embargo, en el 2004, Fawzi realizó un estudio en Tanzania, con 1.078 mujeres embarazadas, positivas para $\mathrm{VIH}$; el objetivo era determinar los efectos del suplemento de vitamina $A$ (vitamina $A$ preformada $y$ $\beta$-carotenos), multivitaminas (vitaminas $B, C$ y E) o ambos sobre la progresión de la enfermedad por el $\mathrm{VIH}$. Estas mujeres se dividieron en cuatro grupos así: Vitamina $A$ (30 mg, $5.000 \mathrm{UI}$ vitamina A preformada), multivitaminas (B1, B2, B3, B6, B12, C, E y folatos), multivitaminas más vitamina $A$ y placebo. Se encontró que en el grupo que recibió multivitaminas, disminuyeron la velocidad de progresión de la enfermedad, los síntomas orales y gastrointestinales, y la carga viral; además, se logró aumentar el recuento de linfocitos T CD4 [18].

Cobre: El cobre es un inhibidor pasivo del VIH, puesto que bloquea la activación intracelular (esencial para la proteasa); su deficiencia aumenta la replicación viral que lleva a un aumento de la progresión de la enfermedad y disminuye el conteo de linfocitos T CD4 [19]. 


\section{Discusión}

El concepto según el cual un paciente desnutrido, debido a estados prolongados de inanición o a diversas alteraciones metabólicas, presenta un profundo estado de inmunosupresión, está universalmente aceptado; pero aún hoy es difícil demostrar cómo las deficiencias específicas de nutrientes contribuyen a un peor desenlace clínico en los pacientes infectados por $\mathrm{VIH}[20,21]$.

Según distribución de sexo y edad, podemos observar que existe una mayor prevalencia en el sexo masculino con un $68 \%$ del total de la muestra, existiendo una razón $\mathrm{H} / \mathrm{M}$ de 2,125; y el 40,8\% para ambos sexos pertenecen al grupo de 18 a 29 años (joven), seguida de cerca con el 39,8\% del grupo de 30 a 35 (adulto joven). Según la situación de VIH/ SIDA en el Perú de acuerdo al Boletín epidemiológico Mensual (2016) [22, 23].

Al analizar los resultados podemos observar que el 48,5\% de nuestra población sujeta a estudio está con un peso 'normal' y un 30,1\% por encima del peso normal (sobrepeso, obesidad 1 y obesidad 2) según el IMC lo que puede generar una incógnita ¿Cómo es posible que un paciente con diagnóstico VIH/SIDA pueda tener un peso normal y/o sobrepeso?; cabe indicar, que el Virus de la inmunodeficiencia adquirida causante del SIDA; está asociada con la pérdida de peso por malnutrición [24].

Con mucha periodicidad se ha podido ver que se asocia a enfermedades de la colágena y con déficit del sistema inmunológico por lo que se usa como tratamiento efectivo los antivirales y los inmunoreguladores del sistema inmunológico, dando mas posibilidades de vida a estas personas, viéndose menos complicaciones en el curso de esta patología [25].

En la medida que vamos equilibrando la alimentación de los pacientes que sufren esta enfermedad, vemos que aumenta la calidad de vida de los mismos y así logramos extender más la vida de los pacientes que sufren este mal, entonces debemos educar a la población en general y en especial a los enfermos, para que conozcan cuales son estos alimentos beneficiosos que favorecen el estado de salud en estos pacientes [26, 27].

Para los hábitos alimentarios el 100\% de nuestra población tienen una sobre adecuación de proteína, probablemente por el sesgo de memoria al momento de la entrevista con el paciente, sin embargo, encontramos un porcentaje alto en referencia al consumo de glúcidos y lípidos; ya que en nuestra sociedad por nuestra misma cultura culinaria y alimenticia se consume más carbohidratos refinados y grasas saturadas [28].

\section{Conclusiones}

La deficiencia de micronutrientes influye en el sistema inmune, acentuando la inmunodeficiencia que lleva al $\mathrm{VIH}$ - Sida. Entre los factores que agravan y pueden acelerar la progresión del estado de la persona que tiene $\mathrm{VIH}$-Sida, se han considerado tres factores nutricionales como los principales: El deficiente aporte nutricional, la deficiente absorción y las alteraciones metabólicas. 
La inmunología nutricional se ve comprometida por la deficiencia de micronutrientes, si se tiene en cuenta que éstos juegan un papel importante en mantener la función inmune normal. El aporte complementario de micronutrientes se ha relacionado con aumento del recuento de linfocitos T CD4, aumento del peso corporal, mejoría del estado clínico, disminución del riesgo de enfermedades oportunistas, aumento de la respuesta linfocitaria a mitógenos con estabilización de la carga viral y disminución del riesgo de muerte.

Aún no se tienen datos exactos sobre las recomendaciones de micronutrientes en pacientes con $\mathrm{VIH} /$ sida; por esta razón, se debe garantizar una dieta balanceada que contenga los micronutrientes recomendados para la población saludable, los cuales ofrecen un tratamiento seguro y económico para retardar la progresión de la infección por el VIH a SIDA.

\section{Conflictos de Intereses}

Los autores no refieren conflictos de intereses.

\section{References}

[1] SIDA. National Geographic. 2019

[2] ONUSIDA/99. 32S. Prevención de la transmisión maternoinfantil del VIH. 1999 October. Available from: http://scielo.sld.cu/scielo.php?pid=S0375-07602000000300012\&script=sci_arttext\&tlng=pt3.

[3] VIH-SIDA. MedlinePlus. 2019 April 29. Available from: https://medlineplus.gov/spanish/hivaids.html

[4] José C.¿Cómo se transmite el VIH? STOPSIDA. 2019 March 28. Available from: https:// masmorbomenosriesgo.es/2019/03/28/como-se-transmite-el-vih-2/

[5] Programa Mundial de Alimentos. Manual de alimentación y nutrición para el cuidado y apoyo de personas adultas viviendo con VIH/SIDA. Bogotá/Colombia D.C.: 2010.

[6] Ministerio de la Protección Social. Guía para el manejo de VIH/Sida basada en la evidencia. Bogotá: 2005. p. 38.

[7] Datos sobre VIH y SIDA. Síntomas, prevención y tratamiento. Plannedparenthood.org. 2019. Available from: https://www.plannedparenthood.org/es/temas-de-salud/ enfermedades-de-transmision-sexual-ets/vih-sida

[8] ¿Qué es la HAART? Drugabuse gov. 2019. Available from: https://www.drugabuse.gov/es/ publicaciones/serie-de-reportes/el-vihsida/que-es-la-haart.

[9] Kotler DP. Management of nutritional alterations and issues concerning quality of life. J Acquir Immune Defic Syndr. 1997;16(Suppl. 1):S30-S35.

[10] Sepúlveda RtT Watson RR. Treatment of antioxidant deficiencies in AIDS patients. Nutr Research. 2002;22:27-37.

[11] Kennedy C, Kuhn L, Stein Z. Vitamin A and HIV infection: disease progression, mortality, and transmission. Nutr Rev. 2000;58:291-303.

[12] Tang AM, Graham NMH, Semba RD, et al. Association between serum vitamin A and E levels and HIV-1 disease progression. AIDS. 1997;11:613-20.

[13] Wang Y, Huang DS, Eskelson CD, et al. Normalization and restoration of nutritional status and immune functions by vitamin E supplementation in murine AIDS. J Nutr. 1994;124:2024-32.

[14] Coovadia H, Bobat R. Zinc deficiency and supplementation in HIV/AIDS. Nutr Research. 2002;22:179-91.

[15] Coovadia H, Bobat R. Zinc deficiency and supplementation in HIV/AIDS. Nutr Research. 2002;22:179-91.

[16] Kupka R, Fawzi W. Zinc nutrition and HIV Infection. Nutr Rev. 2002;50:69-79.

[17] Patrick L. Nutrients and HIV: Part two vitamins A and E, zinc, Bvitamins and magnesium. Altern Med Rev. 2000;5:39-51.

[18] Fawzi W, Msamanga G, Spiegelman D, et al. A randomized trial of multivitamin supplements and HIV disease progression and mortality. N Engl J Med. 2004;351:23-32. 
[19] Semba RD, Graham NMH, Caiaffa WT, et al. Increased mortality associated with vitamin A deficiency during human immunodeficiency virus type 1 infection. Arch Intern Med. 1993;153:2149-54.

[20] Paton NI, Macallan DC, Jebb SA, et al. Longitudinal changes in body composition measured with a variety of methods in patients with AIDS. J Acquir Immune Defic Syndr. 1997;14: 119-127.

[21] Nicholas S, Leung J, Fennoy I. Guidelines for nutritional support of HIV-infected children. J Pediatrics 1991; 119: 59S-62S.

[22] Tang AM, Lanzilloti J, Hendricks K, et al. Micronutrients: Currrent concepts for HIV care providers. AIDS. 2005;19:847-61.

[23] Mahan LK, Escott S, Raymond JL. Krause Dietoterapia. $12^{\text {th }}$ ed. Barcelona-España: Elsevier Masson; 2009.

[24] Villarroya BI. Encuesta dietética, valoración del estado de nutrición e inmunológico de pacientes infectados por el VIH [Tesis Doctoral]. Universidad de Zaragoza; 2009.

[25] Llerena RG, Toledano GM, Figueroa TA, et al. Enfermedades reumáticas y complicaciones metabólicas en pacientes con VIH / SIDA con tratamiento antirretroviral de alta eficiencia. Disponible en: http: //scielo.sld.cu/scielo.php?script=sci_arttext\&pid=S1817-59962018000300005

[26] Jiménez. Expósito M.Tdx.cat. 2019. Available from: https://www.tdx.cat/bitstream/handle/10803/8833/ LIBRO.pdf;sequence=1

[27] Ruesga E, Mosqueda O, Molina L. Disponible en: http://www.revinfcientifica.sld.cu/index.php/ric/article/ view/167/1425

[28] Instituto Nacional de Salud. Centro Nacional de Alimentación y Nutrición Tablas Peruanas de Composición de Alimentos. Lima: Lima Ministerio de Salud; 2009.

[29] datos y cifras del VIH. Organización Mundial de la Salud. 2019 July 19. Available from: https://www. who.int/es/news-room/fact-sheets/detail/hiv-aids

[30] Ministerio de Salud Pública. VIH. Available from: https://www.salud.gob.ec/vih/ 